\title{
Sobolev Spaces on Locally Compact Abelian Groups: Compact Embeddings and Local Spaces
}

\author{
Przemysław Górka, ${ }^{1}$ Tomasz Kostrzewa, ${ }^{1}$ and Enrique G. Reyes ${ }^{2}$ \\ ${ }^{1}$ Department of Mathematics and Information Sciences, Warsaw University of Technology, Ul. Koszykowa 75, 00-662 Warsaw, Poland \\ ${ }^{2}$ Departamento de Matemática y Ciencia de la Computación, Universidad de Santiago de Chile, Casilla 307 Correo 2, Santiago, Chile \\ Correspondence should be addressed to Przemysław Górka; pgorka@mini.pw.edu.pl
}

Received 23 May 2013; Accepted 20 December 2013; Published 9 February 2014

Academic Editor: Kehe Zhu

Copyright ( 2014 Przemysław Górka et al. This is an open access article distributed under the Creative Commons Attribution License, which permits unrestricted use, distribution, and reproduction in any medium, provided the original work is properly cited.

\begin{abstract}
We continue our research on Sobolev spaces on locally compact abelian (LCA) groups motivated by our work on equations with infinitely many derivatives of interest for string theory and cosmology. In this paper, we focus on compact embedding results and we prove an analog for LCA groups of the classical Rellich lemma and of the Rellich-Kondrachov compactness theorem. Furthermore, we introduce Sobolev spaces on subsets of LCA groups and study its main properties, including the existence of compact embeddings into $L^{p}$-spaces.
\end{abstract}

\section{Introduction}

In this paper we continue our research on Sobolev spaces on locally compact abelian groups $[1,2]$, and we examine analogs of the Rellich lemma and the Rellich-Kondrachov compactness theorem. Sobolev spaces are well understood on domains of $\mathbb{R}^{n}$; see $[3,4]$, compact Riemannian manifolds $[5,6]$, and metric measure spaces [7-9]. There are also some works on Sobolev spaces in the $p$-adic context; see $[10,11]$ and references therein and in special cases of locally compact groups such as the Heisenberg group [12].

We are interested in Sobolev spaces in this general context due to our work on nonlinear equations "in infinitely many derivatives" of interest for contemporary physical theories: in [13-15], two of the present authors in collaboration with $\mathrm{H}$. Prado have investigated the existence of regular solutions to the generalized Euclidean Bosonic string equation

$$
\Delta e^{-c \Delta} \phi=U(x, \phi), \quad c>0
$$

and some of its generalizations, and, in $[16,17]$, the same researchers have developed a functional calculus appropriate for the study of the initial value problem for "ordinary" equations of the form

$$
f\left(\partial_{t}\right) \phi=J(t) .
$$

Equations such as (1) and (2) are specially interesting for string theory and cosmology; see [18-21] and references therein. These two areas are undergoing such a fast development that it seems important to understand (1) and (2) in contexts beyond the usual geometric arena of analysis on (Riemannian) manifolds. We think that topological groups are a natural testing ground for gathering a better understanding of (1) and (2). For instance, this setting would allow us to consider (1) for functions $\phi$ on finite spaces with group structure (see, e.g., [22]), or for functions depending on an infinite number of independent variables. On the other hand, this generalization makes it necessary to develop a theory of Sobolev spaces on LCA groups appropriate for the study of nonlocal equations along the lines of [13-15]. It is indeed possible to do so, essentially because of the existence of group structure and the availability of Fourier transform.

We introduced Sobolev spaces on LCA groups in [1]. In that reference, we proved analogs of the Sobolev embedding and Rellich-Kondrachov theorems, and we used these results to prove the existence of regular solutions to (1) on compact abelian groups. Then in [2], we considered a version of the classical Rellich lemma and presented another theorem on regular solutions to (1). Now, our version of the Rellich lemma appearing in [2] relies on a technical assumption on the structure of the group of characters of the given 
group $G$ which limits its applicability. In this paper, we remove this assumption and prove a version of the Rellich lemma which can be applied in great generality, and we also improve our original Rellich-Kondrachov theorem proven in [1]. Moreover, we introduce Sobolev spaces on subsets of LCA groups, in analogy with the Sobolev spaces defined on domains of $\mathbb{R}^{n}$. As in this classical case, we expect these spaces to be useful in the study of differential equations and other applications [23].

We organize this paper as follows. In Section 2, we recall our definition of Sobolev spaces and our previous embedding and compactness results. In Section 3, we state and prove our new compactness results, and in Section 4, we discuss Sobolev spaces on subsets of LCA groups.

We use standard notations from harmonic analysis [24, 25]. Let us fix a locally compact abelian group $G$. We denote by $d x$ the unique Haar measure of $G$ and by $G^{\wedge}$ the dual group of the group $G$; that is, $G^{\wedge}$ is the locally compact abelian group of all continuous group homomorphisms from $G$ to the circle group $T$. The $L^{p}$ spaces over $G$ are defined as usual:

$$
L^{p}(G)=\left\{f: G \longrightarrow \mathbb{C}: \int_{G}|f(x)|^{p} d x<\infty\right\},
$$

and we set

$$
\|f\|_{L^{p}(G)}=\left(\int_{G}|f(x)|^{p} d x\right)^{1 / p} .
$$

We also recall that the Fourier transform on $G$ is defined as follows: if $f \in L^{1}(G)$, then its Fourier transform is the function $\widehat{f}: G^{\wedge} \rightarrow \mathbb{C}$ given by

$$
\widehat{f}(\xi)=\int_{G} \overline{\xi(x)} f(x) d x .
$$

We consider general LCA groups in Section 2, but we restrict ourselves to compact abelian groups when proving compactness results in Section 3.

\section{Sobolev Spaces}

We introduce Sobolev spaces following our previous papers $[1,2]$. Our definition uses essentially the Fourier transform for LCA groups and, as explained in [1], it generalizes naturally the standard notions of Sobolev spaces in the case of $\mathbb{T}^{n}$ and $\mathbb{R}^{n}$; see [26] and [4, Chapter 4].

We denote by $\Gamma$ the set

$$
\begin{gathered}
\Gamma=\left\{\gamma: G^{\wedge} \longrightarrow[0, \infty): \exists_{c_{\gamma}} \forall_{\alpha, \beta \in G^{\wedge}} \gamma(\alpha \beta)\right. \\
\left.\leq c_{\gamma}[\gamma(\alpha)+\gamma(\beta)]\right\} .
\end{gathered}
$$

Definition 1. Let us fix a map $\gamma \in \Gamma$ and a nonnegative real number $s$. We will say that $f \in L^{2}(G)$ belongs to the Sobolev space $H_{\gamma}^{s}(G)$ if the following integral is finite:

$$
\int_{G^{\wedge}}\left(1+\gamma(\xi)^{2}\right)^{s}|\widehat{f}(\xi)|^{2} d \xi
$$

Moreover, for $f \in H_{\gamma}^{s}(G)$, its norm $\|f\|_{H_{\gamma}^{s}(G)}$ is defined as follows:

$$
\|f\|_{H_{\gamma}^{s}(G)}=\left(\int_{G^{\wedge}}\left(1+\gamma(\xi)^{2}\right)^{s}|\widehat{f}(\xi)|^{2} d \xi\right)^{1 / 2} .
$$

Remark 2. A particular instance of Definition 1 appears in the paper [26] by Feichtinger and Werther. Another particular case of our definition is in [27]. We also note that in $p$-adic analysis, Sobolev spaces are defined in a way analogous to our Definition 1: if we take $\gamma(\xi)=\|\xi\|_{p}$, where $\|\cdot\|_{p}$ is a $p$-adic norm on $\mathbb{Q}_{p}^{n} \simeq \mathbb{Q}_{p}^{n \wedge}$, then (7) and (8) allow us to recover the $p$-adic Sobolev spaces considered in [11].

Remark 3. The fact that $\gamma \in \Gamma$ implies that our spaces $H_{\gamma}^{s}(G)$ are Banach algebras under some assumptions on $s$; see our previous paper [1].

We recall the following results proven in [1].

Proposition 4. Let $G$ be a locally compact abelian group. One has the following.

(1) Consider $H_{\gamma}^{s}(G) \hookrightarrow L^{2}(G)$. Moreover, for each $f \in$ $H_{\gamma}^{s}(G)$, the following inequality holds:

$$
\|f\|_{L^{2}(G)} \leq\|f\|_{H_{\gamma}^{s}(G)} .
$$

(2) If $s>\sigma$, then $H_{\gamma}^{s}(G) \hookrightarrow H_{\gamma}^{\sigma}(G)$. Moreover, the following inequality holds:

$$
\|f\|_{H_{\gamma}^{\sigma}(G)} \leq\|f\|_{H_{\gamma}^{s}(G)} .
$$

Theorem 5. Let $G$ be a locally compact abelian group. One has the following.

(1) If $1 /\left(1+\gamma(\cdot)^{2}\right) \in L^{s}\left(G^{\wedge}\right)$, then

$$
H_{\gamma}^{s}(G) \hookrightarrow C(G),
$$

in which $C(G)$ denotes the space of continuous complexvalued functions on $G$. Moreover, there exists a constant $C(\gamma, s)$ such that for each $f \in H_{\gamma}^{s}(G)$, the following inequality holds:

$$
\|f\|_{C(G)} \leq C(\gamma, s)\|f\|_{H_{\gamma}^{s}(G)} .
$$

(2) If $\alpha>s$ and $1 /\left(1+\gamma(\cdot)^{2}\right) \in L^{\alpha}\left(G^{\wedge}\right)$, then

$$
H_{\gamma}^{s}(G) \hookrightarrow L^{\alpha^{*}}(G),
$$

where $\alpha^{*}=2 \alpha /(\alpha-s)$. Moreover, there exists a constant $D(\gamma, s)$ such that for each $f \in H_{\gamma}^{s}(G)$, the following inequality holds:

$$
\|f\|_{L^{\alpha^{*}(G)}} \leq D(\gamma, s)\|f\|_{H_{\gamma}^{s}(G)} .
$$

Theorem 5 is our version of the classical Sobolev embedding theorem appearing, for instance, in [3] for the case $G=$ $\mathbb{R}^{n}$. 


\section{Compact Embedding}

We recall that the notation $A \hookrightarrow \hookrightarrow B$ means that the space $A$ is compactly embedded in $B$. We begin with our refined version of the classical Rellich lemma.

Theorem 6. Let $G$ be a compact group. If $\lim _{\xi \rightarrow \infty} \gamma(\xi)=\infty$, that is, for each $M>0$ there exists finite set $U$ such that for any $\xi \in G^{\wedge} \backslash U$, one has $\gamma(\xi) \geq M$, then for all $s>\delta$,

$$
H_{\gamma}^{s}(G) \hookrightarrow \hookrightarrow H_{\gamma}^{\delta}(G) .
$$

Proof. We begin the proof stating a classical fact (see, e.g., [28]) on the characterization of precompact sets in $L^{p}(H)$ spaces.

Theorem 7 (see [28]). Let $\Phi$ be a family of functions in $L^{p}(H)$, $1 \leq p<\infty$. Then $\Phi$ is compact in $L^{p}(H)$ if and only if the following conditions hold.

(i) There exists $M>0$ such that $\|\phi\|_{L^{p}(H)} \leq M$ for all $\phi \in \Phi$.

(ii) For every $\varepsilon>0$, there exists compact set $K$ in $G$ such that for each $\phi \in \Phi$,

$$
\|\phi\|_{L^{p}(H \backslash K)} \leq \varepsilon .
$$

(iii) For all $\varepsilon>0$, there exists unit neighborhood $V$ such that for all $x \in V$ and all $\phi \in \Phi$,

$$
\left\|L_{x} \phi-\phi\right\|_{L^{p}(H)} \leq \varepsilon .
$$

Now we return to the proof of Theorem 6. Let $f_{n}$ be a bounded sequence in $H_{\gamma}^{s}(G),\left\|f_{n}\right\|_{H_{\gamma}^{s}(G)} \leq M$. We need to show that there exists subsequence that converges strongly in $H_{\gamma}^{\delta}(G)$. We will prove this fact by showing that the following sequence

$$
g_{n}(\xi)=\left|\widehat{f}_{n}(\xi)\right|\left(1+\gamma^{2}(\xi)\right)^{\delta / 2}
$$

is compact in $L^{2}\left(G^{\wedge}\right)$. We use Weil's theorem: since $\delta<s$, we get

$$
\left\|g_{n}\right\|_{L^{2}\left(G^{\wedge}\right)} \leq\left\|f_{n}\right\|_{H_{\gamma}^{s}(G)} \leq M,
$$

and so (i) holds. Now we consider condition (ii). Let us fix $\epsilon>0$. We consider two cases: if $G^{\wedge}$ is finite group, then we can take simply $K=G^{\wedge}$ and condition (ii) is satisfied. On the other hand, if the dual group $G^{\wedge}$ is infinite, it is enough to recall that if $G$ is a compact group, then by the Pontryagin duality theorem, its dual $G^{\wedge}$ is discrete and therefore every compact set must be finite. From our assumption, we can find a compact set $K$ such that

$$
\frac{1}{\left(1+\gamma^{2}(\xi)\right)^{s-\delta}} \leq \frac{\epsilon}{M^{2}}, \quad \text { for all } \xi \in G^{\wedge} \backslash K .
$$

Hence,

$$
\begin{aligned}
& \int_{G^{\wedge} \backslash K}\left|\widehat{f}_{n}(\xi)\right|^{2}\left(1+\gamma^{2}(\xi)\right)^{\delta} d \xi \\
& \quad=\int_{G^{\wedge} \backslash K}\left|\widehat{f}_{n}(\xi)\right|^{2} \frac{\left(1+\gamma^{2}(\xi)\right)^{s}}{\left(1+\gamma^{2}(\xi)\right)^{s-\delta}} d \xi \\
& \quad \leq \sup _{\xi \in G^{\wedge} \backslash K} \frac{1}{\left(1+\gamma^{2}(\xi)\right)^{s-\delta}} \int_{G^{\wedge} \backslash K}\left|\widehat{f}_{n}(\xi)\right|^{2}\left(1+\gamma^{2}(\xi)\right)^{s} d \xi \\
& \quad \leq\left\|f_{n}\right\|_{H_{\gamma}^{s}(G)}^{2} \sup _{\xi \in G^{\wedge} \backslash K} \frac{1}{\left(1+\gamma^{2}(\xi)\right)^{s-\delta}} \leq \epsilon,
\end{aligned}
$$

and so (ii) holds. It remains to check condition (iii). Since $G^{\wedge}$ is discrete and each set is open, we can take $V=\{e\}$, where $e$ is unit in $G^{\wedge}$. Thus, condition (iii) is satisfied and Theorem 6 follows from Weil's result.

Theorem 6 appears in our previous paper [2] under the additional assumption that the dual group $G^{\wedge}$ is countable.

Now we consider embeddings of $H_{\gamma}^{s}(G)$ into $C(G)$ and $L^{p}(G)$. We proved in [1] that $H_{\gamma}^{s}(G)$ is continuously embedded in $C(G)$. We prove in Theorem 10 below that if $G$ is compact, then $H_{\gamma}^{s} \hookrightarrow \hookrightarrow C(G)$, and finally in Theorem 11, we consider a version of the Rellich-Kondrachov which refines an analogous result from [1]. We need the following lemma.

Lemma 8. Let $H$ be a discrete group and $f \in L^{1}(H)$. Then for every $\varepsilon>0$, there exists a finite set $U$ such that for any $h \in H \backslash U$, one has $|f(h)| \leq \varepsilon$.

Proof. Let us suppose that the theorem is not true, so that there is a number $\varepsilon>0$ such that for every finite set $U$, there exists $h \in H \backslash U$ with $|f(h)| \geq \varepsilon$. Let $U_{1}$ be a finite set and let $h_{1} \in H \backslash U_{1}$ be such that $\left|f\left(h_{1}\right)\right| \geq \varepsilon$. We define $U_{2}=U_{1} \cup\left\{h_{1}\right\}$. Since $U_{2}$ is finite, there exists $h_{2} \in H \backslash U_{2}$ such that $\left|f\left(h_{2}\right)\right| \geq \varepsilon$. By induction, we get a sequence of sets $\left\{U_{i}\right\}$ and $\left\{h_{i}\right\}$ for $i=1,2, \ldots$ such that $U_{i+1}=U_{i} \cup\left\{h_{i}\right\}$ and for each $i$, we have $\left|f\left(h_{i}\right)\right| \geq \varepsilon$. Since Haar measure on discrete groups is a multiple of the counting measure, we get

$$
\begin{aligned}
\int_{H}|f(h)| d h & =\int_{H \backslash U}|f(h)| d h+\int_{U}|f(h)| d h \\
& \geq \sum_{i=1}^{\infty}\left|f\left(h_{i}\right)\right| \geq \sum_{i=1}^{\infty} \varepsilon=+\infty,
\end{aligned}
$$

where $U=\bigcup_{i=1}^{\infty} U_{i}$, and this is a contradiction.

Remark 9. We note that if $H$ is countable, the proof of Lemma 8 is elementary: $\int_{H}|f(h)| d h=a \sum_{i=1}^{\infty}\left|f\left(h_{i}\right)\right|$ for some $a \in \mathbb{C}$ and $\sum_{i=1}^{\infty}\left|f\left(h_{i}\right)\right|<\infty$ implies that given $\epsilon>0$, there exists $K>0$ such that $\sum_{i=K}^{\infty}\left|f\left(h_{i}\right)\right|<\epsilon$. The result then follows.

Theorem 10. If $G$ is compact, $1 /\left(1+\gamma^{2}(\cdot)\right) \in L^{\alpha}\left(G^{\wedge}\right)$, and $s<\alpha$ then

$$
H_{\gamma}^{s}(G) \hookrightarrow \hookrightarrow C(G)
$$


Proof. Using Lemma 8 for the function $1 /\left(1+\gamma^{2}(\cdot)\right)^{\alpha}$, we see that $\gamma$ satisfies the assumptions of Theorem 6 , so for $s<\alpha$, we get that

$$
H_{\gamma}^{s}(G) \hookrightarrow \hookrightarrow H_{\gamma}^{\alpha}(G)
$$

Moreover, thanks to the first part of Theorem 5, we have

$$
H_{\gamma}^{\alpha}(G) \hookrightarrow C(G),
$$

and the proof follows.

Theorem 11. If $G$ is compact, $1 /\left(1+\gamma^{2}(\cdot)\right) \in L^{\alpha}\left(G^{\wedge}\right)$, and $s<\alpha$, then

$$
H_{\gamma}^{s}(G) \hookrightarrow \hookrightarrow L^{p}(G)
$$

for all $p<\alpha^{*}=2 \alpha /(\alpha-s)$.

Proof. Let us take $\delta=\alpha-2 \alpha / p$; then, $\delta<s$. Thus, by Theorem 6 , we have the compact embedding

$$
H_{\gamma}^{s}(G) \hookrightarrow \hookrightarrow H_{\gamma}^{\delta}(G)
$$

Next, using the second part of Theorem 5, we have the continuous embedding

$$
H_{\gamma}^{\delta}(G) \hookrightarrow L^{\alpha_{\delta}^{*}}(G)
$$

where $\alpha_{\delta}^{*}=2 \alpha /(\alpha-\delta)=p$.

\section{Sobolev Spaces on Subsets of LCA Groups}

In this section, we deal with Sobolev spaces defined on subsets of locally compact abelian groups. As mentioned in Section 1, we are motivated by analogous studies of function spaces on domains of $\mathbb{R}^{n}$ (see, e.g., [29]) by the fact that interesting applications exist, [23], and by the possibility of using them as tools for the study of differential equations on subsets of LCA groups. We start with the following definition.

Definition 12. Let $U$ be a subset of a LCA group $G$. We define the Sobolev space $H_{\gamma}^{s}(U) \subset L^{2}(U)$ as the space of all $f \in$ $L^{2}(U)$ such that there exists $F \in H_{\gamma}^{s}(G)$ with $\left.F\right|_{U}=f$ and we equip it with the norm

$$
\|f\|_{H_{\gamma}^{s}(U)}=\inf \left\{\|F\|_{H_{\gamma}^{s}(G)}: F \in H_{\gamma}^{s}(G) \text { and }\left.F\right|_{U}=f\right\} \text {. }
$$

An analogous definition (of spaces $B_{p q}^{s}$ on domains of $\mathbb{R}^{n}$ ) appears in [29]; see his Definition 2.3. It can be easily shown that $H_{\gamma}^{s}(U)$ is a Banach space. We will say that it is a local Sobolev space.

Using appropriate embeddings for $H_{\gamma}^{s}(G)$ and the definition of $H_{\gamma}^{s}(U)$, we can prove the following.

Theorem 13. Let $G$ be a locally compact abelian group and let $U \subset G$. Then we have.
(1) The continuous inclusion $H_{\gamma}^{s}(U) \hookrightarrow L^{2}(U)$ holds. Moreover, for each $f \in H_{\gamma}^{s}(U)$, the following inequality holds:

$$
\|f\|_{L^{2}(U)} \leq\|f\|_{H_{\gamma}^{s}(U)} .
$$

(2) If $s>\sigma$, then $H_{\gamma}^{s}(G) \hookrightarrow H_{\gamma}^{\sigma}(U)$. Moreover, the following inequality holds:

$$
\|f\|_{H_{\gamma}^{\sigma}(U)} \leq\|f\|_{H_{\gamma}^{s}(U)} .
$$

(3) If $1 /\left(1+\gamma(\cdot)^{2}\right) \in L^{s}\left(G^{\wedge}\right)$, then $H_{\gamma}^{s}(U) \hookrightarrow C(U)$. Moreover, there exists a constant $C(\gamma, s)$ such that for each $f \in H_{\gamma}^{s}(U)$, the following inequality holds:

$$
\|f\|_{C(U)} \leq C(\gamma, s)\|f\|_{H_{\gamma}^{s}(U)} .
$$

(4) If $\alpha>s$ and $1 /\left(1+\gamma(\cdot)^{2}\right) \in L^{\alpha}\left(G^{\wedge}\right)$, then $H_{\gamma}^{s}(U) \hookrightarrow$ $L^{\alpha^{*}}(U)$, where $\alpha^{*}=2 \alpha /(\alpha-s)$. Moreover, there exists a constant $D(\gamma, s)$ such that for each $f \in H_{\gamma}^{s}(G)$, the following inequality holds:

$$
\|f\|_{L^{\alpha^{*}}(U)} \leq D(\gamma, s)\|f\|_{H_{\gamma}^{s}(U)} .
$$
detail

We now prove the following compactness theorem in

Theorem 14. Let $G$ be an LCA group and let $U$ be a subset of $G$ of finite measure. Assume that $1 /\left(1+\gamma^{2}(\cdot)\right) \in L^{\alpha}\left(G^{\wedge}\right)$ for some $\alpha>s$ and that

$$
\frac{|\xi(h)-1|}{\left(1+\gamma^{2}(\xi)\right)^{s}} \underset{h \rightarrow e}{\longrightarrow} 0 \text { uniformly with respect to } \xi \in G^{\wedge}
$$

Then, for all $p<\alpha^{*}$, one has the compact embedding

$$
H_{\gamma}^{s}(U) \hookrightarrow \hookrightarrow L^{p}(U)
$$

The convergence concept used in (34) is explained in our previous paper [1]. Let us mention that a condition similar to (34) appears in the characterization of precompact sets in $L^{2}(G)$ via Fourier transform; see [30].

Proof. We will need two lemmas which we proved in [1].

Lemma 15. Let $F \in H_{\gamma}^{s}(G)$ and assume that

$$
\frac{|\xi(h)-1|}{\left(1+\gamma^{2}(\xi)\right)^{s}} \underset{h \rightarrow e}{\longrightarrow} 0 \text { uniformly with respect to } \xi \in G^{\wedge}
$$

Then, for every $h \in G$,

$$
\int_{G}|F(x h)-F(x)|^{2} d x \leq C(h)\|F\|_{H_{\gamma}^{s}(G)}^{2},
$$

where $C(h) \underset{h \rightarrow e}{\rightarrow} 0$. 
Lemma 16. Let us denote by $\mathscr{I}$ the set of all symmetric unit neighbourhoods of group $G$ partially ordered by inclusion. Let $\left(\phi_{V}\right)_{V \in \mathcal{F}}$ be a Dirac net and let $F \in H_{\gamma}^{s}(G)$. Then,

$$
\int_{G}\left|F * \phi_{V}(x)-F(x)\right|^{2} d x \leq\|F\|_{H_{\gamma}^{s}(G)} \sup _{y \in V} C(y) .
$$

Now we can continue with the proof of Theorem 14. Let $f_{n}$ be a bounded sequence in $H_{\gamma}^{s}(U)$; that is, $\left\|f_{n}\right\|_{H_{\gamma}^{s}(U)} \leq C$. From the very definition, there exist functions $F_{n}$ such that $F_{n}=f_{n}$ in $U, F_{n} \in H_{\gamma}^{s}(G)$ and $\left\|F_{n}\right\|_{H_{\gamma}^{s}(G)} \leq\left\|f_{n}\right\|_{H_{\gamma}^{s}(U)}+1$ for $n=1,2, \ldots$. Now, by Theorem 13, we get that the sequence $f_{n}$ is bounded in $L^{\alpha^{*}}(U)$. Hence, there exists a subsequence $f_{n_{k}}$ such that $f_{n_{k}} \rightarrow f$ in $L^{\alpha^{*}}(U)$ and there also exists a subsequence $F_{n_{k}}$ such that $F_{n_{k}} \rightarrow F$ in $L^{\alpha^{*}}(G)$. For simplicity, we simply write $f_{n}$ and $F_{n}$ for these subsequences. From the basic properties of weak convergence, we have that $F=f$ in $U$. We will show that for $q<\alpha^{*}$, we have

$$
f_{n} \longrightarrow f \text { in } L^{q}(U) .
$$

Let us set $F_{(V)}=F * \phi_{V}$. From the triangle inequality, we get

$$
\begin{aligned}
\left\|f_{n}-f\right\|_{L^{2}(U)} \leq & \left\|f_{n}-F_{n(V)}\right\|_{L^{2}(U)} \\
& +\left\|F_{n(V)}-F_{(V)}\right\|_{L^{2}(U)}+\left\|F_{(V)}-f\right\|_{L^{2}(U)} \\
\leq & \left\|F_{n}-F_{n(V)}\right\|_{L^{2}(G)}+\left\|F_{n(V)}-F_{(V)}\right\|_{L^{2}(U)} \\
& +\left\|F_{(V)}-F\right\|_{L^{2}(G)} .
\end{aligned}
$$

From Lemma 15, we get

$$
\begin{aligned}
\sup _{n} \int_{G}\left|F_{n_{(V)}}(x)-F_{n}(x)\right|^{2} d x & \leq \sup _{n}\left\|F_{n}\right\|_{H_{\gamma}^{s}(G)}^{2} \sup _{y \in V} C(y) \\
& \leq(C+1)^{2} \sup _{y \in V} C(y) .
\end{aligned}
$$

Furthermore it can be easily shown that for each $\epsilon>0$, there exists $V_{\epsilon}$ such that for all $V \in \mathscr{I}$ with $V \subset V_{\epsilon}$, we have

$$
\left\|F_{(V)}-F\right\|_{L^{2}(G)} \leq \frac{\epsilon}{3} .
$$

Hance, we can choose $V_{\epsilon}$ such that

$$
\left\|f_{n}-f\right\|_{L^{2}(U)} \leq \frac{2}{3} \epsilon+\left\|F_{n\left(V_{\epsilon}\right)}-F_{\left(V_{\epsilon}\right)}\right\|_{L^{2}(U)} .
$$

We need to estimate $\left\|F_{n\left(V_{\epsilon}\right)}-F_{\left(V_{\epsilon}\right)}\right\|_{L^{2}(U)}$. Since $F_{n} \rightarrow F$ in $L^{\alpha^{*}}(G)$, we get that $F_{n\left(V_{\epsilon}\right)}(x) \underset{n \rightarrow \infty}{\rightarrow} F_{\left(V_{\epsilon}\right)}(x)$. Moreover, we have

$$
\begin{aligned}
& \left|F_{n\left(V_{\epsilon}\right)}(x)-F_{\left(V_{\epsilon}\right)}(x)\right|^{2} \\
& \quad=\left|\int_{G}\left(F_{n}(y)-F(y)\right) \phi_{V_{\epsilon}}\left(y^{-1} x\right) d y\right|^{2}
\end{aligned}
$$

$$
\begin{aligned}
= & \left|\int_{G}\left(F_{n}(y)-F(y)\right) \sqrt{\phi_{V_{\varepsilon}}\left(y^{-1} x\right)} \sqrt{\phi_{V_{\varepsilon}}\left(y^{-1} x\right)} d y\right|^{2} \\
\leq & \int_{G} \phi_{V_{\epsilon}}\left(y^{-1} x\right) d \mu_{G}(y) \\
& \times \int_{G}\left|F_{n}(y)-F(y)\right|^{2} \phi_{V_{\varepsilon}}\left(y^{-1} x\right) d y \\
\leq & \sup _{z \in V_{\varepsilon}} \phi_{V_{\epsilon}}(z) \int_{G}\left|F_{n}(y)-F(y)\right|^{2} \phi_{V_{\varepsilon}}\left(y^{-1} x\right) d y .
\end{aligned}
$$

Hence, since $U$ has a finite measure, we get from Lebesgue convergence theorem that

$$
\left\|F_{n\left(V_{\epsilon}\right)}-F_{\left(V_{\epsilon}\right)}\right\|_{L^{2}(U)} \leq \frac{\epsilon}{3} .
$$

We conclude that $f_{n} \rightarrow f$ in $L^{2}(U)$. In order to finish the proof, it is enough to use the Vitali convergence theorem. We conclude that $f_{n} \rightarrow f$ in $L^{q}(U)$ for $q<\alpha^{*}$.

\section{Conflict of Interests}

The authors declare that there is no conflict of interests regarding the publication of this paper.

\section{Acknowledgments}

Przemysław Górka's research is partially supported by the European Union in the framework of European Social Fund through the Warsaw University of Technology Development Program. Enrique G. Reyes' research is partially supported by the Project FONDECYT no. 1111042.

\section{References}

[1] P. Górka and E. G. Reyes, "Sobolev spaceson locally compact abelian groups," http://arXiv.org/abs/1208.3053, in press.

[2] P. Górka, T. Kostrzewa, and E. G. Reyes, "The Rellich lemma on compact abelian groups and equations of infinite order," International Journal of Geometric Methods in Modern Physics, vol. 10, no. 2, Article ID 1220030, 11 pages, 2013.

[3] R. A. Adams, Sobolev Spaces, Academic Press, New York, NY, USA, 1975.

[4] M. E. Taylor, Partial Differential Equations, vol. 23, Springer, New York, NY, USA, 1996.

[5] T. Aubin, Some Nonlinear Problems in Riemannian Geometry, Springer, Berlin, Germany, 1998.

[6] E. Hebey, Sobolev Spaces on Riemannian Manifolds, vol. 1635, Springer, Berlin, Germany, 1996.

[7] P. Hajłasz, "Sobolev spaces on an arbitrary metric space," Potential Analysis, vol. 5, no. 4, pp. 403-415, 1996.

[8] P. Hajłasz and P. Koskela, "Sobolev met Poincaré," Memoirs of the American Mathematical Society, vol. 145, no. 688, 2000.

[9] N. Shanmugalingam, "Newtonian spaces: an extension of Sobolev spaces to metric measure spaces," Revista Matemática Iberoamericana, vol. 16, no. 2, pp. 243-279, 2000. 
[10] Y.-C. Kim, "A simple proof of the $p$-adic version of the Sobolev embedding theorem," Korean Mathematical Society, vol. 25, no. 1, pp. 27-36, 2010.

[11] J. J. Rodríguez-Vega and W. A. Zúñiga-Galindo, "Elliptic pseudodifferential equations and Sobolev spaces over $p$-adic fields," Pacific Journal of Mathematics, vol. 246, no. 2, pp. 407-420, 2010.

[12] H. Bahouri, C. Fermanian-Kammerer, and I. Gallagher, "Analyse de l'espace des phases et calcul pseudo-differential sur le groupe de Heisenberg," Comptes Rendus Mathématique, vol. 347, no. 17-18, pp. 1021-1024, 2009.

[13] P. Górka, H. Prado, and E. G. Reyes, "Nonlinear equations with infinitely many derivatives," Complex Analysis and Operator Theory, vol. 5, no. 1, pp. 313-323, 2011.

[14] P. Górka, H. Prado, and E. G. Reyes, "Generalized euclidean bosonic string equations," in Operator Theory: Advances and Applications, vol. 224, pp. 147-169, Springer, Basel, Switzerland, 2012.

[15] P. Górka, H. Prado, and E. G. Reyes, "On a general class of nonlocal equations,” Annales Henri Poincaré, vol. 14, no. 4, pp. 947-966, 2013.

[16] P. Górka, H. Prado, and E. G. Reyes, "Functional calculus via Laplace transform and equations with infinitely many derivatives," Journal of Mathematical Physics, vol. 51, no. 10, Article ID 103512, 2010.

[17] P. Górka, H. Prado, and E. G. Reyes, "The initial value problem for ordinary differential equations with infinitely many derivatives," Classical and Quantum Gravity, vol. 29, no. 6, Article ID 065017, 2012.

[18] N. Barnaby and N. Kamran, "Dynamics with infinitely many derivatives: the initial value problem," Journal of High Energy Physics, no. 2, 40 pages, 2008.

[19] G. Calcagni, M. Montobbio, and G. Nardelli, "Localization of nonlocal theories," Physics Letters B, vol. 662, no. 3, pp. 285-289, 2008.

[20] V. S. Vladimirov, "On the equation of a $p$-adic open string for a scalar tachyon field," Izvestiya, vol. 69, no. 3, pp. 487-512, 2005.

[21] E. Witten, "Noncommutative geometry and string field theory," Nuclear Physics B, vol. 268, no. 2, pp. 253-294, 1986.

[22] D. Zhou and C. J. C. Burges, "High-order regularization on graphs," in Proceedings of the 6th International Workshop on Mining and Learning with Graphs, Helsinki, Finland, 2008, http://www.cis.hut.fi/MLG08/papers/postersession/paper39.pdf.

[23] H. Triebel, "Wavelets in function spaces on Lipschitz domains," Mathematische Nachrichten, vol. 280, no. 9-10, pp. 1205-1218, 2007.

[24] E. Hewitt and K. A. Ross, Abstract Harmonic Analysis. Vol. I, Die Grundlehren der mathematischen Wissenschaften, Springer, Berlin, Germany, 1963.

[25] E. Hewitt and K. A. Ross, Abstract Harmonic Analysis. Vol. II, Die Grundlehren der mathematischen Wissenschaften, Springer, Berlin, Germany, 1970.

[26] H. G. Feichtinger and T. Werther, "Robustness of regular sampling in Sobolev algebras," in Sampling, Wavelets, and Tomography, J. Benedetto and A. I. Zayed, Eds., pp. 83-113, Birkhäuser Boston, Boston, Mass, USA, 2004.

[27] E. Popescu, "Nonlocal Dirichlet forms generated by pseudodifferential operators on compact abelian groups," Proceedings in Applied Mathematics and Mechanics, vol. 7, pp. 21600012160002, 2007.

[28] A. Weil, L'Intégration dans les Groupes Topologiques et ses Applications, Hermann et Cie, Paris, France, 1940.
[29] H. Triebel, "Function spaces in Lipschitz domains and on Lipschitz manifolds. Characteristic functions as pointwise multipliers," Revista Matemática Complutense, vol. 15, no. 2, pp. 475-524, 2002.

[30] P. Górka, "Pego theorem on locally compact abelian groups," Journal of Algebra and Its Applications, vol. 13, no. 4, Article ID 1350143, 2014. 


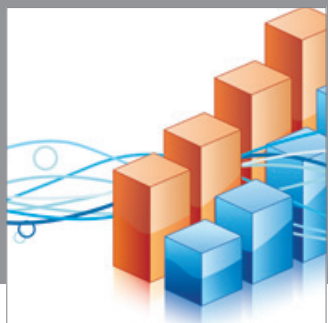

Advances in

Operations Research

mansans

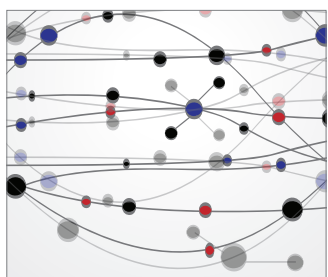

The Scientific World Journal
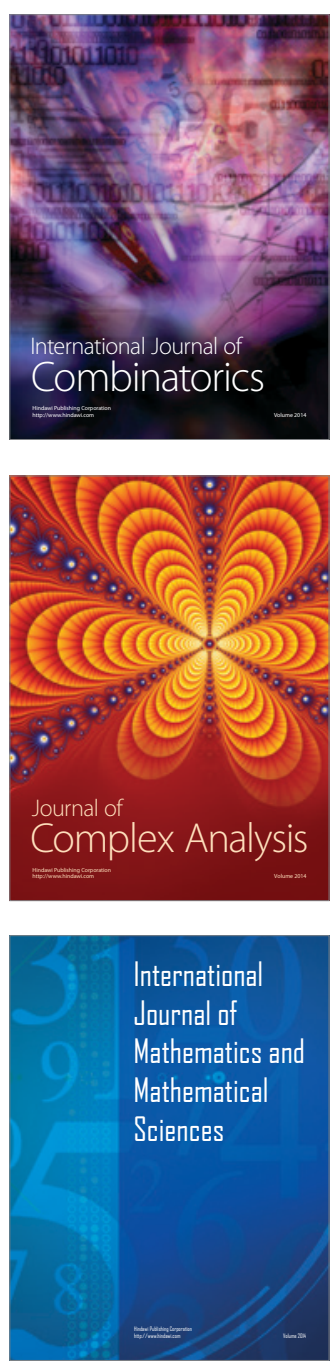
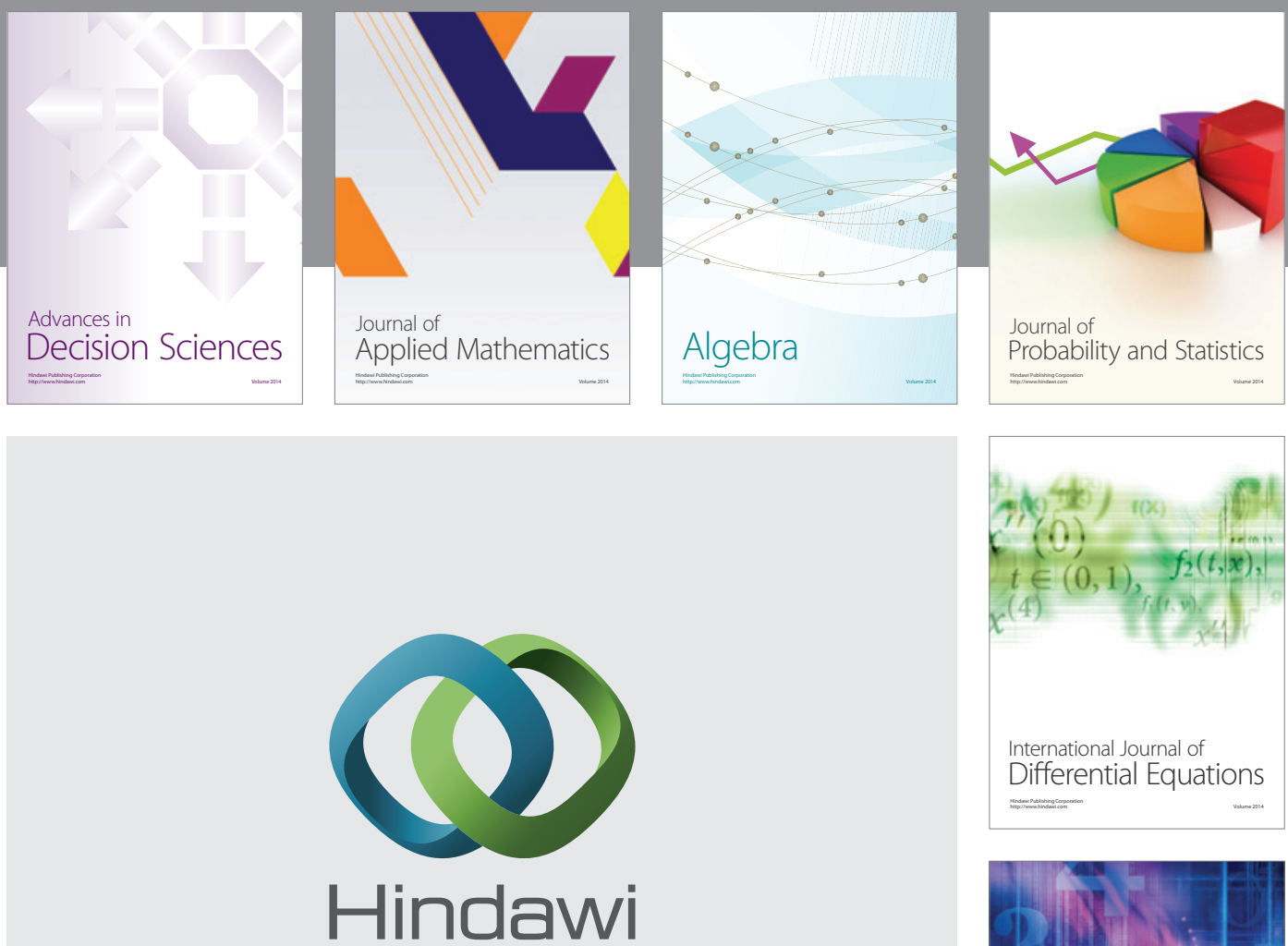

Submit your manuscripts at http://www.hindawi.com
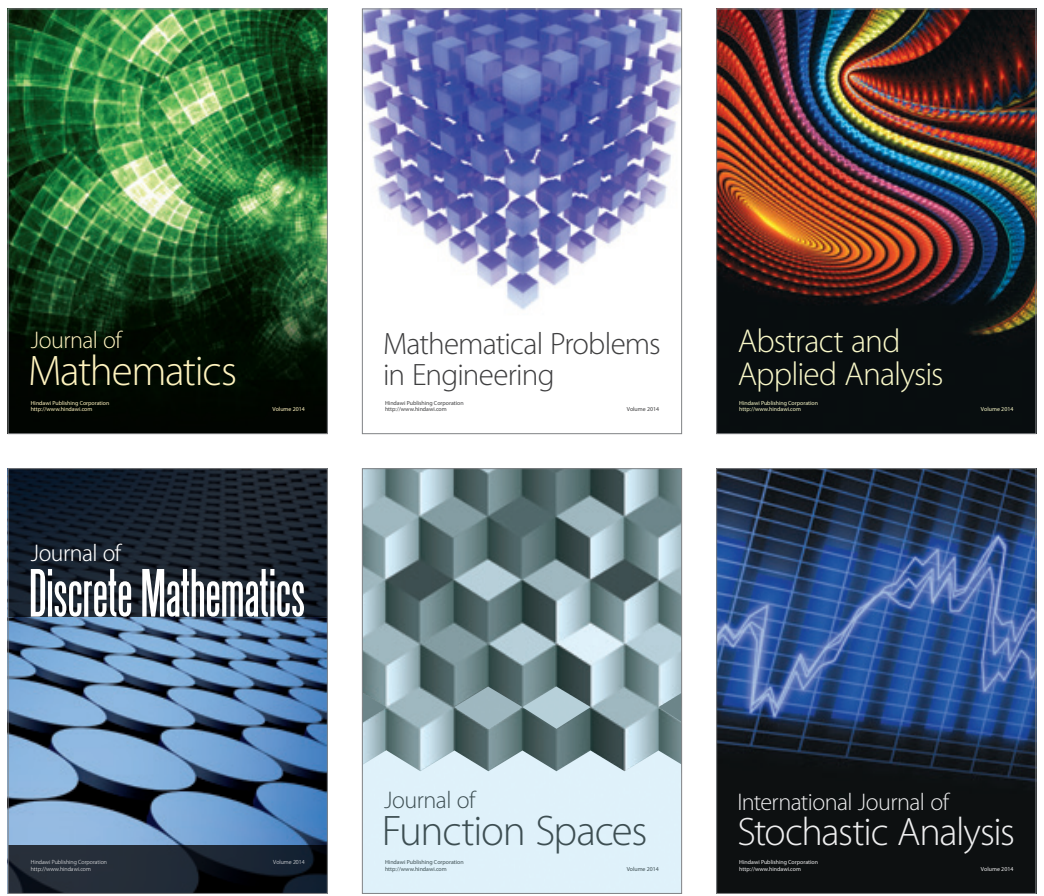

Journal of

Function Spaces

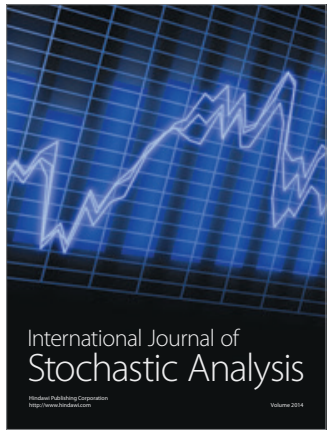

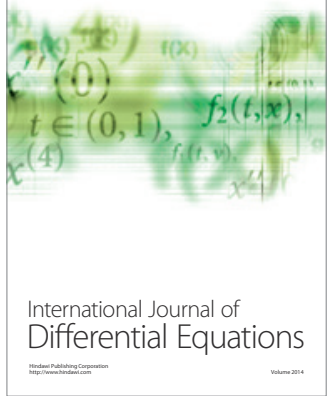
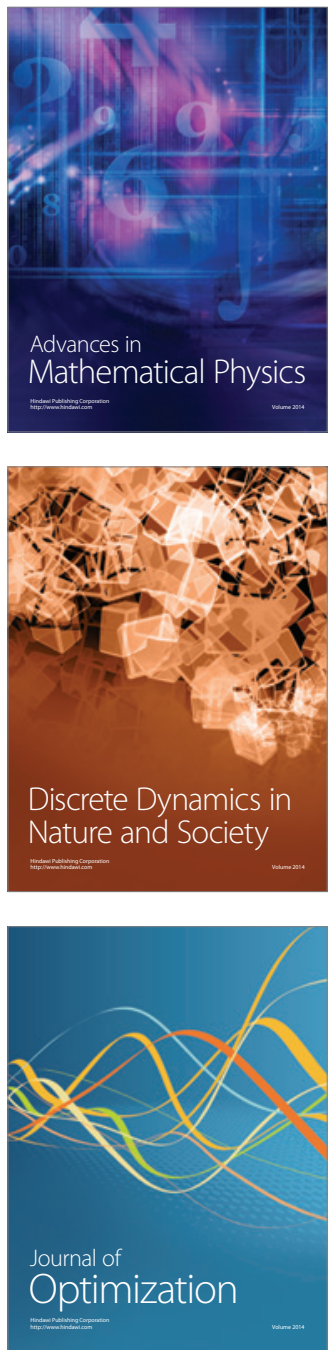